\title{
Multigrid Method for Poroelasticity Problem by Finite Element Method
}

\author{
Luoping Chen ${ }^{1}$ and Yanping Chen ${ }^{2, *}$ \\ ${ }^{1}$ School of Mathematics, Southwest Jiaotong University, Chengdu 611756, Sichuan, \\ China \\ 2 School of Mathematical Sciences, South China Normal University, \\ Guangzhou 510631, Guangdong, China
}

Received 6 January 2019; Accepted (in revised version) 31 March 2019

\begin{abstract}
In this paper, we will investigate a multigrid algorithm for poroelasticity problem by a new finite element method with homogeneous boundary conditions in two dimensional space. We choose Nédélec edge element for the displacement variable and piecewise continuous polynomials for the pressure variable in the model problem. In constructing multigrid algorithm, a distributive Gauss-Seidel iteration method is applied. Numerical experiments shows that the finite element method achieves optimal convergence order and the multigrid algorithm is almost uniformly convergent to mesh size $h$ and parameter $\delta t$ on regular meshes.
\end{abstract}

AMS subject classifications: 65M10, 78A48

Key words: Poroelasticity problem, finite element method, multigrid method.

\section{Introduction}

General theory describing the consolidation of a porous elastic soil is very important in application, for example, predicting the behavior of foundation resting on a saturated clay is an important problem in foundation engineering. The foundation allows for the occurrence of finite geometry changes and finite elastic strains during the consolidation process. This theory of poroelasticity addresses the time-dependent coupled process between the deformation of porous materials and the fluid flow inside. The governing equations have been cast in a rate form and laws which determine deformation and pore fluid flow are Hookes's law and Darcy's law. The theoretical basis of consolidation was established by Terzaghi [22], then, Biot generalized the theory to three dimensional transient consolidation $[4,5]$. Since then, poroelastic theory has been used in a diverse range

*Corresponding author.

Emails: cherrychen@home.swjtu.edu.cn (L. P. Chen), yanpingchen@scnu.edu.cn (Y. P. Chen) 
of science and engineering application, for instance, $\mathrm{CO}_{2}$ sequestration in environmental engineering [14] is important applications of poroelasticity. Recently, research in poroelasticity has been a surge in activity, not only because of the application described above, but also due to emerging applications in biomechanics engineering such as biological soft tissue modeling including arterial walls, skin, cardiac muscle and articular cartilage $[12,15,20,25]$.

There are extensive literatures on numerical methods for poroelasticity. The most commonly used numerical discretization are finite element methods for the two fields problem, for example, a continuous Galerkin (CG) element for both displacement and pressure is studied in $[13,16]$. Later, finite element method based on three fields (displacement, fluid flux, pore pressure) are analyzed, for instance, couple continuous and discontinuous Galerkin (DG) methods for the displacement with a mixed finite element method for the flow variables are investigated in [17-19]. Also, Yi [27] studied the nonconforming finite element for displacement and standard mixed finite element method for pressure and velocity and Tchonkova [21] used a mixed finite element method which seeks a solution via minimization of a least-squares functionals. Four fields formulation with displacement, stress, pressure and flux is also studied in [26]. Meanwhile, finite difference method on staggered mesh [7], finite volume method [2] for the problem with discontinuous coefficients and weak Galerkin method [10] are also considered for the poroelasticity model problems.

As mesh size becomes much smaller, the scale of discrete equation of poroelasticity model by numerical methods becomes much larger. Therefore, efficient solver for the discrete linear system is important in computing numerical solution. There are lots of studies in constructing fast solvers for poroelascitity model problem, see examples $[1,3,6,9,11$, 24]. Multigrid algorithm is one of the most efficient iterative scheme which can reduce the computation of discrete equation to $\mathcal{O}(N)$ or $\mathcal{O}(N \log N)$ with $N$ being the scale of the linear system. For the poroelasticity problem, some efficient multigrid algorithm has been designed based on finite difference method. For instance, multigrid algorithm with distributive smoothing on cartesian equidistant grids are constructed in $[8,24]$. Performance of multigrid methods with two different types of smoother, the decouple smoother (distributive type) and couple smoother (Vanka type) are compared for poroelasticity model in [9] on staggered mesh. In this work, we will study the multigrid method with decoupled smoother by finite element method on triangular mesh.

We will investigate the finite element method as well as the multigrid method for poroelasticity model problem in this study. There are mainly two unknowns, displacement variable $\boldsymbol{u}$ and pore pressure $p$ in the poroelasticity model problems. We constructed the discretization method by introduce an intermediate variable $\omega=-\operatorname{div} u$. For the discrete finite element spaces, we choose the Nédélec edge element for the displacement variable and piecewise continuous Lagrange polynomials for the intermediate variable $\omega$ as well as for pore pressure variable. Numerical results show that on regular mesh, this discretization method achieves the optimal order in $L^{2}$ norm and corresponding energy norms. We also constructed the multigrid algorithm for the poroelasticity 
problem based on this finite element method. We mainly concern using the distributive Gauss Seidel iteration as smoother in the multigrid algorithm. Numerical experiments shows that the $\mathrm{W}$-cycle multigrid algorithm with both two-step pre- and post-smoothing is a uniform convergent algorithm to both mesh size $h$ and parameter $\delta t$ (which relates to time step size in the model problem).

The outline of the paper is as follows. In Section 2, we will introduce the model problem, weak formulation and the finite element method. Multigrid algorithm with distributive Gauss Seidel iteration for the discrete system will be constructed in Section 3. In Section 4, we implement two examples and show the performance of the finite element method as well as the multigrid algorithm numerically.

\section{Model problem and finite element method}

Mechanical processes in poroelasticity mainly contain two basic element: fluid flow and deformation of elasticity body. Mathematical model for the poroelasticity are derived from physical principles, i.e., the mass conservation and momentum balance for both fluid and elasticity body. The momentum balance for the solid phase is give as the following well known equilibrium conditions for the stress tensor field $\tilde{\sigma}$ :

$$
\operatorname{div} \tilde{\sigma}=-f,
$$

where $f$ representing the external force acting on the domain $\Omega$.

From the poroelasticity theory, the effective stress $\sigma$ is defined as

$$
\sigma=\tilde{\sigma}+c_{\text {up }} p \boldsymbol{I},
$$

with $c_{u p}$ being a positive coefficient, $p$ being a fluid pressure and $I$ is the identity matrix. Let $\boldsymbol{u}$ denote the displacement variable and $\boldsymbol{\epsilon}(\boldsymbol{u})$ be the strain tensor, the classical straindisplacement relation is defined as

$$
\boldsymbol{\epsilon}(\boldsymbol{u})=\frac{1}{2}\left(\nabla \boldsymbol{u}+(\nabla \boldsymbol{u})^{T}\right) .
$$

The relation between effective stress and train tensor is presented as follows

$$
\sigma=2 \mu \boldsymbol{\epsilon}(\boldsymbol{u})+\lambda \operatorname{tr}(\boldsymbol{\epsilon}(\boldsymbol{u})) \boldsymbol{I},
$$

with $\lambda, \mu$ being Láme coefficients and tr being the trace operator.

We have the following relation

$$
\begin{aligned}
\operatorname{div} \tilde{\sigma} & =-f \Leftrightarrow \operatorname{div}\left(\sigma-c_{u p} p \cdot \boldsymbol{I}\right)=-f \\
& \Leftrightarrow-\operatorname{div}(2 \mu \boldsymbol{\epsilon}+\lambda \operatorname{tr} \boldsymbol{\epsilon} \cdot \boldsymbol{I})+\operatorname{div} c_{u p} p \cdot \boldsymbol{I}=\boldsymbol{f} \\
& \Leftrightarrow-2 \mu \operatorname{div} \boldsymbol{\epsilon}+\lambda \operatorname{div}(\operatorname{tr}(\boldsymbol{\epsilon}) \cdot \boldsymbol{I})+\operatorname{div} c_{u p} p \cdot \boldsymbol{I}=\boldsymbol{f} \\
& \Leftrightarrow-2 \mu \operatorname{div} \boldsymbol{\epsilon}+\lambda \operatorname{div}(\operatorname{tr}(\boldsymbol{\epsilon}) \cdot \boldsymbol{I})+\operatorname{div} c_{u p} p \cdot \boldsymbol{I}=\boldsymbol{f} \\
& \Leftrightarrow-\mu \operatorname{div} \operatorname{grad} \boldsymbol{u}-\mu \operatorname{div}(\operatorname{grad} \boldsymbol{u})^{T}-\lambda \operatorname{grad} \operatorname{div} \boldsymbol{u}+c_{u p} \operatorname{grad} p=\boldsymbol{f} \\
& \Leftrightarrow-\mu \operatorname{div} \operatorname{grad} \boldsymbol{u}-(\lambda+\mu) \operatorname{grad} \operatorname{div} \boldsymbol{u}+c_{u p} \operatorname{grad} p=\boldsymbol{f} .
\end{aligned}
$$


Denote $\theta$ as the increment of fluid volume per unit volume of soil, then,

$$
\theta=c_{p u} \operatorname{div} \boldsymbol{u}+c_{p p} p,
$$

the momentum balance of fluid satisfies the Darcy law

$$
\boldsymbol{q}=-K \nabla p
$$

and the mass conservative law of the incompressible fluid is presented as

$$
\frac{\partial \theta}{\partial t}=-\operatorname{div} \boldsymbol{q}+S_{f}
$$

where $S_{f}$ representing the flow rate from an external source.

From (2.5)-(2.7), we have the following relation

$$
\frac{\partial\left(c_{p u} \operatorname{div} u\right)}{\partial t}+\frac{\partial\left(c_{p p} p\right)}{\partial t}+\operatorname{div}(-K \nabla p)=S_{f}
$$

Finally, poroelasticity model problem is described as

$$
\left\{\begin{array}{l}
-\mu \operatorname{divgrad} \boldsymbol{u}-(\lambda+\mu) \operatorname{grad} \operatorname{div} \boldsymbol{u}+c_{u p} \operatorname{grad} p=-f \\
\frac{\partial\left(c_{p u} \operatorname{div} \boldsymbol{u}\right)}{\partial t}+\frac{\partial\left(c_{p p} p\right)}{\partial t}+\operatorname{div}(-K \nabla p)=S_{f} .
\end{array}\right.
$$

General boundary condition of this system is represented as

$$
p=0 \quad \text { on } \Gamma_{p}, \nabla p \cdot \boldsymbol{n}=0 \quad \text { on } \Gamma_{f}, \quad \boldsymbol{u}=0 \quad \text { on } \Gamma_{d}, \boldsymbol{\sigma} \cdot \boldsymbol{n}=\boldsymbol{t} \quad \text { on } \Gamma_{t},
$$

with $\partial \Omega=\Gamma_{p} \cup \Gamma_{f}$ for pressure variable and $\partial \Omega=\Gamma_{d} \cup \Gamma_{t}$ for displacement variable. The initial condition is

$$
\operatorname{div} \boldsymbol{u}(0)=0 \quad \text { in } \Omega .
$$

For simplicity, in this paper, we will consider the model problem with $c_{u p}=c_{p u}=1$, $c_{p p}=0$ and $K=\kappa / \eta$ in the system, where $\kappa$ is the permeability of the porous skeleton and $\eta$ is the viscosity of the pore fluid. The existence and uniqueness of the solution of this model problem was proved by Ženišek [23]. In this work, we mainly consider constructing uniformly convergent multigrid algorithm, therefore, we will confine our study in numerical solution of model problem with fixed time, i.e., we treat time step size $\delta t$ as a parameter in the problem. We investigate the edge finite element method and multigrid algorithm for following system,

$$
\begin{aligned}
& -\mu \boldsymbol{\Delta} \boldsymbol{u}-(\lambda+\mu) \nabla \nabla \cdot \boldsymbol{u}+\nabla p=\boldsymbol{f}, \\
& \nabla \cdot \boldsymbol{u}-\frac{\kappa}{\eta} \delta t \Delta p=g,
\end{aligned}
$$


with $\delta t$ being some constant related to the time step size. We also restrict the discussion of the discretization method and multigrid method with homogeneous Dirichlet boundary condition

$$
p=0, \quad \boldsymbol{u}=0 \quad \text { on } \partial \Omega,
$$

which corresponding to $\Gamma_{f}=\Gamma_{t}=0$.

We first introduce some notations and spaces that used in this study. We denote $(\cdot, \cdot)$ as the inner product in $L^{2}(\Omega)$ and $W^{m, q}$ as standard Sobolev space with norm $\|\cdot\|_{m, q}$ given by $\|v\|_{m, q}^{q}=\sum_{\alpha \leq m}\left\|D^{\alpha} v\right\|_{L^{q}(\Omega)}^{q}$. For $q=2$, we let $H^{m}(\Omega)=W^{m, 2}(\Omega),\|\cdot\|_{m}=\|\cdot\|_{m, 2}$ and $\|\cdot\|=\|\cdot\|_{0,2} \cdot H_{0}^{1}(\Omega)$ be the subspace of $H^{1}(\Omega)$ with homogenous boundary condition. Let

$$
\begin{aligned}
& \boldsymbol{H}(\operatorname{rot})=\left\{\boldsymbol{v} \mid \boldsymbol{v} \in\left(L^{2}(\Omega)\right)^{2}, \operatorname{rot} \boldsymbol{v} \in L^{2}(\Omega)\right\}, \\
& \boldsymbol{H}_{0}(\operatorname{rot})=\{\boldsymbol{v} \mid \boldsymbol{v} \in \boldsymbol{H}(\operatorname{rot}), \boldsymbol{v} \times \boldsymbol{n}=0\},
\end{aligned}
$$

with $n$ being the normal vector of the boundary. Differential operators of grad,div and rot that used in the content are defined as follows

$$
\operatorname{grad} q=\left(\frac{\partial q}{\partial x}, \frac{\partial q}{\partial y}\right)^{t}, \quad \operatorname{curl} q=\left(\frac{\partial q}{\partial y},-\frac{\partial q}{\partial x}\right)^{t}, \quad \operatorname{div} v=\frac{\partial v_{1}}{\partial x}+\frac{\partial v_{2}}{\partial y}, \quad \operatorname{rot} v=\frac{\partial v_{2}}{\partial x}-\frac{\partial v_{1}}{\partial y},
$$

with $t$ on top right corner representing the transpose of a vector or matrix.

It is easy to check that the vector Laplace operator can be represented as

$$
-\Delta=\text { curlrot }- \text { graddiv } \text {. }
$$

We first introduce the intermediate variable $\omega=-\operatorname{div} \boldsymbol{u}$, then, (2.10) can be presented as following differential system

$$
\begin{aligned}
& \omega+\operatorname{div} \boldsymbol{u}=0, \\
& \mu \text { curlrot } \boldsymbol{u}+(\lambda+2 \mu) \operatorname{grad} \omega+\operatorname{grad} p=\boldsymbol{f}, \\
& \operatorname{div} \boldsymbol{u}-\delta t \frac{\kappa}{\eta} \operatorname{divgrad} p=g .
\end{aligned}
$$

The weak form of the system can be represented as: find $(\omega, \boldsymbol{u}, p) \in H^{1}(\Omega) \times \boldsymbol{H}(\operatorname{rot}) \times$ $H^{1}(\Omega)$ such that

$$
\begin{cases}-(\omega, \tau)+(\boldsymbol{u}, \nabla \tau)=(\boldsymbol{u} \cdot \boldsymbol{n}, \tau)_{\partial \Omega}, & \forall \tau \in H^{1}(\Omega), \\ (\mu \nabla \times \boldsymbol{u}, \nabla \times \boldsymbol{v})+((\lambda+2 \mu) \nabla \omega, \boldsymbol{v})+(\nabla p, \boldsymbol{v})=(\boldsymbol{f}, \boldsymbol{v})-(\nabla \times \boldsymbol{u} \times \boldsymbol{n , v})_{\partial \Omega}, & \forall \boldsymbol{v} \in \boldsymbol{H}(\operatorname{rot}), \\ (\boldsymbol{u}, \nabla q)-\left(\delta t \frac{\kappa}{\eta} \nabla p, \nabla q\right)=-(g, q)+(\boldsymbol{u} \cdot \boldsymbol{n}, q)_{\partial \Omega}-(\nabla p \cdot \boldsymbol{n}, q)_{\partial \Omega,} & \forall q \in H^{1}(\Omega) .\end{cases}
$$

Recall the boundary condition in the model, we have

$$
\boldsymbol{u}=0 \quad \text { on } \partial \Omega \Leftrightarrow \boldsymbol{u} \cdot \boldsymbol{n}=0 \quad \text { and } \quad \boldsymbol{u} \times \boldsymbol{n}=0 \quad \text { on } \partial \Omega .
$$


The normal boundary condition of $\boldsymbol{u}$ is enforced weakly by dropping the right hand side in the first equation of (2.11) and the tangential boundary condition $\boldsymbol{u} \times \boldsymbol{n}=0$ is enforce in the strong sense, i.e., $\boldsymbol{u} \in \boldsymbol{H}_{0}$ (rot). We enforce the boundary condition of $p$ in the strong sense too, i.e., $p \in H_{0}^{1}(\Omega)$. Then, the weak formulation (2.11) is presented to find $(\omega, \boldsymbol{u}, p) \in H^{1}(\Omega) \times \boldsymbol{H}_{0}(\operatorname{rot}) \times H_{0}^{1}(\Omega)$ such that

$$
\begin{cases}-(\omega, \tau)+(\boldsymbol{u}, \nabla \tau)=0, & \forall \tau \in H^{1}(\Omega), \\ (\mu \nabla \times \boldsymbol{u}, \nabla \times \boldsymbol{v})+((\lambda+2 \mu) \nabla \omega, \boldsymbol{v})+(\nabla p, \boldsymbol{v})=(\boldsymbol{f}, \boldsymbol{v}), & \forall \boldsymbol{v} \in \boldsymbol{H}_{0}(\operatorname{rot}), \\ (\boldsymbol{u}, \nabla q)-\left(\delta t \frac{\kappa}{\eta} \nabla p, \nabla q\right)=-(g, q), & \forall q \in H_{0}^{1}(\Omega) .\end{cases}
$$

For the discrete form, we use the piecewise continuous polynomial to approximate Sobolev spaces $H^{1}(\Omega), H_{0}^{1}(\Omega)$ and Nédélec edge element to approximate the displacement space. Suppose $\mathbb{V}_{h}, \mathbb{N}_{h}, \mathbb{V}_{h}^{0}$ represent the discrete finite element spaces of $H^{1}(\Omega)$, $\boldsymbol{H}_{0}(\operatorname{rot}), H_{0}^{1}(\Omega)$, respectively. The discrete weak formulation of the system (2.12) can be represented as finding $\left(\omega_{h}, \boldsymbol{u}_{h}, p_{h}\right) \in \mathbb{V}_{h} \times \mathbb{N}_{h} \times \mathbb{V}_{h}^{0}$ such that,

$$
\begin{cases}-\left(\omega_{h}, \tau\right)+\left(\boldsymbol{u}_{h}, \nabla \tau\right)=0, & \forall \tau \in \mathbb{V}_{h} \\ \left(\mu \nabla \times \boldsymbol{u}_{h}, \nabla \times \boldsymbol{v}\right)+\left((\lambda+2 \mu) \nabla \omega_{h}, \boldsymbol{v}\right)+\left(\nabla p_{h}, \boldsymbol{v}\right)=(\boldsymbol{f}, \boldsymbol{v}), & \forall \boldsymbol{v} \in \mathbb{N}_{h} \\ \left(\boldsymbol{u}_{h}, \nabla q\right)-\left(\delta t \frac{\kappa}{\eta} \nabla p_{h}, \nabla q\right)=-(g, q), & \forall q \in \mathbb{V}_{h}^{0}\end{cases}
$$

In order to presenting the matrix form of the system, we shall describe the incidence matrix in two-dimension in the following. Suppose $\Omega \subset \mathbb{R}^{2}$ is divided by uniform or quasi uniform triangular mesh with $N_{t}$ elements, $N_{e}$ edges and $N_{v}$ nodes. The cells, edges and nodes of the triangulation $\mathcal{T}$ are denoted generically by $\tau_{i}, \boldsymbol{e}_{j}$ and $v_{k}$. For each edge $\boldsymbol{e}_{j}$, we fix a tangential direction $\boldsymbol{t}_{j}$ and normal direction $\boldsymbol{n}_{j}$, such that $\left(\boldsymbol{n}_{j}, \boldsymbol{t}_{j}\right)$ are oriented like the $(x, y)$ axes of the coordinate system. The $N_{t} \times N_{e}$ element-edge incidence matrix $\mathcal{R}$ and the $N_{e} \times N_{v}$ edge-node incidence matrix $\mathcal{G}$ of the triangulation $\mathcal{T}$ are defined as follows,

$$
\begin{aligned}
& \mathcal{R}_{i, j}:= \begin{cases}+1, & \text { if } \boldsymbol{n}_{j} \text { is directed out of element } \tau_{i}, \\
-1, & \text { if } \boldsymbol{n}_{j} \text { is directed into element } \tau_{i}, \\
0, & \text { if } \boldsymbol{n}_{j} \text { does not meet element } \tau_{i} .\end{cases} \\
& \mathcal{G}_{j, k}:= \begin{cases}+1, & \text { if } \boldsymbol{t}_{j} \text { is directed into node } v_{k}, \\
-1, & \text { if } \boldsymbol{t}_{j} \text { is directed out of node } v_{k}, \\
0, & \text { if } \boldsymbol{t}_{j} \text { does not meet node } v_{k} .\end{cases}
\end{aligned}
$$

Note that $\mathcal{R}$ and $\mathcal{G}$ are discrete version of rot and grad operators without scaling. Then, the continous relation rotgrad $=0$ implies the disrete relationship $\mathcal{R G}=0$. 
Now, the corresponding matrix form of the discrete system is:

$$
\left(\begin{array}{ccc}
-M_{v} & \mathcal{G}^{t} M_{e} & 0 \\
(\lambda+2 \mu) M_{e} \mathcal{G} & \mu \mathcal{R}^{t} M_{t} \mathcal{R} & M_{e} \mathcal{G} \\
0 & \mathcal{G}^{t} M_{e} & -\delta t \frac{\kappa}{\eta} \mathcal{G}^{t} M_{e} \mathcal{G}
\end{array}\right)\left(\begin{array}{l}
W_{h} \\
U_{h} \\
P_{h}
\end{array}\right)=\left(\begin{array}{c}
0 \\
M_{e} f_{I} \\
-M_{v} g
\end{array}\right)
$$

where $M_{v}, M_{e}, M_{t}$ are mass matrices in $H(\operatorname{grad}), H(\operatorname{rot}), L^{2}(\Omega)$ respectively.

Now, denote

$$
B=\mathcal{G}^{t} M_{e} \quad \text { (representing }- \text { div operator), }
$$

and we introduce the following notations as the matrix form of the operator with boundary conditions:

$$
\begin{array}{ll}
\tilde{B}_{0}: & \text { Enforce the boundary condition of } u \text { to } B, \\
B_{0}: & \text { Enforce the boundary condition of } u, p \text { to } B, \\
\mathcal{R}_{0}: & \text { Enforce the boundary condition of } u \text { to incidence matrix } \mathcal{R}, \\
C_{0}: & \text { Enforce the boundary condition of } p \text { to scalar Laplace operator, } \\
\mathcal{G}_{0}: & \text { Enforce the boundary condition of } u, p \text { to the incidence matrix }, \\
M_{v 0}: & \text { Enforce the boundary condition of } \omega \text { to the mass matrix } M_{v} .
\end{array}
$$

Then, after adding the boundary condition and eliminate $W_{h}$ from the first equation of system (2.14), we get the following matrix system

$$
\mathcal{L}=\left(\begin{array}{cc}
(\lambda+2 \mu) \tilde{B}_{0}^{t} M_{v}^{-1} \tilde{B}_{0}+\mu \mathcal{R}_{0}^{t} M_{t} \mathcal{R}_{0} & B_{0}^{t} \\
B_{0} & -\delta t \frac{\kappa}{\eta} C_{0}
\end{array}\right)=\left(\begin{array}{cc}
A & B^{t} \\
B & -A_{p}
\end{array}\right),
$$

where $\mathcal{L}$ being coefficient matrix of the system to displacement unknown vector $U_{h}$ and pressure unknown vector $P_{h}$. $A, A_{p}$ are representing matrix form of vector Laplace and scalar Laplace operators multiplied by some constants, It is obvious that the matrix form of the model problem is a saddle point problem with both vector Laplace and scalar Laplace operator on the diagonals. In the following, we will focus on construct the uniformly convergence multigrid method to mesh size $h$ and time step size $\delta t$ for this system.

\section{Multigrid algorithm based on distributive Gauss Seidel smoother}

The purpose of this section is to construct uniformly convergent multigrid algorithm for the poroelasticity model problem discretized by finite element method discussed in Section 2. As we know, in order to design a uniformly convergent multigrid algorithm, 
smoother is a very important ingredient. In this paper, we mainly study using the decoupled relaxation, i.e., the distributive Gauss-Seidel, as the smoother in the multigrid algorithm.

In order to present the distributive Gauss Seidel relaxation method, we first present the coefficient operator matrix of the system (2.10) as $\mathscr{L}$ in the follows,

$$
\mathscr{L}=\left(\begin{array}{cc}
-\mu \Delta-(\lambda+\mu) \text { graddiv } & \text { grad } \\
-\operatorname{div} & \left(\delta t \frac{\kappa}{\eta}\right) \Delta
\end{array}\right),
$$

and construct the corresponding transform operator as

$$
\mathscr{M}=\left(\begin{array}{cc}
I & \text { grad } \\
(\lambda+\mu) \operatorname{div} & -(\lambda+2 \mu) \operatorname{divgrad}
\end{array}\right),
$$

then, we have

$$
\mathscr{L} \cdot \mathscr{M}=\left(\begin{array}{cc}
-\mu \Delta & 0 \\
-\operatorname{div}+\left(\delta t \frac{\kappa}{\eta}(\lambda+\mu)\right) \Delta \operatorname{div} & -\operatorname{divgrad}+\delta t \frac{\kappa}{\eta}(\lambda+2 \mu) \Delta \text { divgrad }
\end{array}\right) .
$$

As we can see from the continuous operator form, after multiplication by $\mathscr{M}$, the resulting system $\mathscr{L} \cdot \mathscr{M}$ is a block lower triangular system and theoretically we can solve the first equation with displacement variable $\boldsymbol{u}$ only and then solve the second equation for unknown pore pressure $p$ with known displacement $\boldsymbol{u}$.

The matrix form of the transform operator $\mathscr{M}$ is

$$
\overline{\mathcal{M}}=\left(\begin{array}{cc}
I & \mathcal{G} \\
-(\lambda+\mu) M_{v}^{-1} B & -(\lambda+2 \mu) M_{v}^{-1} B \mathcal{G}
\end{array}\right) .
$$

After enforcing the boundary condition, we have transform matrix with boundary as

$$
\begin{aligned}
\mathcal{M} & =\left(\begin{array}{cc}
I & \mathcal{G}_{0} \\
-(\lambda+\mu) M_{v 0}^{-1} B_{0} & -(\lambda+2 \mu) M_{v 0}^{-1} B_{0} \mathcal{G}_{0}
\end{array}\right) \\
& =\left(\begin{array}{cc}
I & M_{12} \\
M_{21} & M_{22}
\end{array}\right) .
\end{aligned}
$$

Then, the transformed system with boundary condition is

$$
\begin{aligned}
\mathcal{L} \mathcal{M} & =\left(\begin{array}{cc}
(\lambda+2 \mu) \tilde{B}_{0}^{t} M_{v}^{-1} \tilde{B}_{0}+\mu \mathcal{R}_{0}^{t} M_{t} \mathcal{R}_{0} & B_{0}^{t} \\
B_{0} & -\frac{\delta t \kappa}{\eta} \mathcal{R}_{0}
\end{array}\right)\left(\begin{array}{cc}
I & \mathcal{G}_{0} \\
-(\lambda+\mu) M_{v 0}^{-1} B_{0} & -(\lambda+2 \mu) M_{v 0}^{-1} B_{0} \mathcal{G}_{0}
\end{array}\right) \\
& \approx\left(\begin{array}{cc}
(\lambda+2 \mu) \tilde{B}_{0}^{t} M_{v}^{-1} \tilde{B}_{0}+\mu \mathcal{R}_{0}^{t} M_{t} \mathcal{R}_{0}-(\lambda+\mu) B_{0}^{t} M_{v 0}^{-1} B_{0} & 0 \\
B_{0}+\frac{\delta t \kappa}{\eta}(\lambda+\mu) C_{0} M_{v 0}^{-1} B_{0} & B_{0} \mathcal{G}_{0}+\frac{\delta t \kappa}{\eta}(\lambda+2 \mu) C_{0} M_{v 0}^{-1} B_{0} \mathcal{G}_{0}
\end{array}\right) .
\end{aligned}
$$


Remark 3.1. The explicit formula of $(1,2)$ block of the matrix $\mathcal{L} \mathcal{M}$ is

$$
(\lambda+2 \mu)\left(\tilde{B}_{0}^{t} M_{v}^{-1} \tilde{B}_{0}-B_{0}^{t} M_{v 0}^{-1} B_{0}\right) \mathcal{G}_{0}+\mu \mathcal{R}_{0}^{t} M_{t} \mathcal{R}_{0} \mathcal{G}_{0} .
$$

If no boundary condition enforced, formula in the bracket of the fist term is exact zero and $\mathcal{R G}=0$, which is the discrete form of rotgrad $=0$. However, after enforcing the boundary condition, the result matrix form approximates to zero and nonzero terms appear on boundary elements of the triangulation.

Remark 3.2. For the $(1,1)$ block of the matrix $\mathcal{L} \mathcal{M}$, as we can see from the expression, $(\lambda+$ $2 \mu) \tilde{B}_{0}^{t} M_{v}^{-1} \tilde{B}_{0}+\mu \mathcal{R}_{0}^{t} M_{t} \mathcal{R}_{0}-(\lambda+\mu) B_{0}^{t} M_{v 0}^{-1} B_{0}$ is exactly the discretization of vector Laplace operator(times by $\mu$ ) in the interior grid. After enforce the boundary condition of $\boldsymbol{u}, p$, this discrete block approximates to the vector Laplace operator, therefore, we will choose smoothers that is efficient for vector Laplace for this term.

Remark 3.3. We also notice that after transformation of the system, the $(2,2)$ block of $\mathcal{L} \mathcal{M}$ is a fourth order operator, which makes the smoothing to this system more complicated than the well known Stokes problem.

Now, denote $\mathcal{T}$ as the approximate transformed system

$$
\begin{aligned}
\mathcal{T} & =\left(\begin{array}{cc}
(\lambda+2 \mu) \tilde{B}_{0}^{t} M_{v}^{-1} \tilde{B}_{0}+\mu \mathcal{R}_{0}^{t} M_{t} \mathcal{R}_{0}-(\lambda+\mu) B_{0}^{t} M_{v 0}^{-1} B_{0} & 0 \\
B_{0}+\delta t \frac{\kappa}{\eta}(\lambda+\mu) C_{0} M_{v 0}^{-1} B_{0} & B_{0} \mathcal{G}_{0}+\frac{\delta t \kappa(\lambda+2 \mu)}{\eta} C_{0} M_{v 0}^{-1} B_{0} \mathcal{G}_{0}
\end{array}\right) \\
& =\left(\begin{array}{cc}
S_{u} & 0 \\
T_{21} & S_{p}
\end{array}\right),
\end{aligned}
$$

with $S_{u}$ being a matrix approximating vector Laplace operator and $S_{p}$ being an matrix of some fourth order operator.

The distributive Gauss Seidel iteration method for this system is represented as follows with initial guess $\left(\boldsymbol{u}^{0}, p^{0}\right)^{t}$,

$$
\left(\begin{array}{l}
\boldsymbol{u}^{k+1} \\
p^{k+1}
\end{array}\right)=\left(\begin{array}{c}
\boldsymbol{u}^{k} \\
p^{k}
\end{array}\right)+\boldsymbol{\omega} \mathcal{M} \mathcal{T}^{-1}\left[\left(\begin{array}{l}
f \\
g
\end{array}\right)-\mathcal{L}\left(\begin{array}{l}
\boldsymbol{u}^{k} \\
p^{k}
\end{array}\right)\right], \quad k=0,1, \cdots,
$$

with $\omega$ being some relaxation parameter.

We then simplify the update process in the iteration by introducing an intermediate step

$$
\left(\begin{array}{l}
\boldsymbol{r}_{u} \\
r_{p}
\end{array}\right)=\left(\begin{array}{l}
f \\
g
\end{array}\right)-\mathcal{L}\left(\begin{array}{c}
\boldsymbol{u}^{k} \\
p^{k}
\end{array}\right)
$$

We split the computation of $\mathcal{M} \mathcal{T}^{-1}$ as

$$
\left(\begin{array}{l}
\boldsymbol{u}^{k+1} \\
p^{k+1}
\end{array}\right)=\left(\begin{array}{c}
\boldsymbol{u}^{k} \\
p^{k}
\end{array}\right)+\omega\left(\begin{array}{cc}
I & 0 \\
M_{21} & 0
\end{array}\right)\left(\begin{array}{c}
S_{u} \backslash \boldsymbol{r}_{u} \\
0
\end{array}\right)+\omega\left(\begin{array}{ll}
0 & M_{12} \\
0 & M_{22}
\end{array}\right)\left(\begin{array}{c}
0 \\
-S_{p}^{-1} T_{21} S_{u}^{-1} \boldsymbol{r}_{u}+S_{p}^{-1} r_{p}
\end{array}\right) .
$$


Denote

$$
\left(\begin{array}{l}
\boldsymbol{u}^{k+\frac{1}{2}} \\
p^{k+\frac{1}{2}}
\end{array}\right)=\left(\begin{array}{c}
\boldsymbol{u}^{k} \\
p^{k}
\end{array}\right)+\omega\left(\begin{array}{c}
S_{u} \backslash \boldsymbol{r}_{u} \\
M_{21}\left(S_{u} \backslash \boldsymbol{r}_{u}\right)
\end{array}\right) .
$$

Notice that

$$
\begin{aligned}
g-B \boldsymbol{u}^{k+\frac{1}{2}}+A_{p} p^{k+\frac{1}{2}} & =-\omega T_{21} S_{u}^{-1} \boldsymbol{r}_{u}+r_{p} \\
& =\omega\left(-T_{21} S_{u}^{-1} \boldsymbol{r}_{u}+r_{p}\right)+\left(r_{p}-\omega r_{p}\right) .
\end{aligned}
$$

We denote

$$
\begin{aligned}
r_{p}^{1} & =g-B \boldsymbol{u}^{k+\frac{1}{2}}+A_{p} p^{k+\frac{1}{2}}-\left(r_{p}-\boldsymbol{\omega} r_{p}\right) \\
& =\omega\left(-T_{21} S_{u}^{-1} \boldsymbol{r}_{u}+r_{p}\right)
\end{aligned}
$$

then

$$
\left(\begin{array}{l}
\boldsymbol{u}^{k+1} \\
p^{k+1}
\end{array}\right)=\left(\begin{array}{l}
\boldsymbol{u}^{k+\frac{1}{2}} \\
p^{k+\frac{1}{2}}
\end{array}\right)+\left(\begin{array}{l}
M_{12}\left(S_{p} \backslash r_{p}^{1}\right) \\
M_{22}\left(S_{p} \backslash r_{p}^{1}\right)
\end{array}\right) .
$$

Detail of the implementation of distributive Gauss Seidel relaxation can be represented as follows:

\section{Algorithm 3.1. Distributive Guass Seidel relaxation.}

Step 1. Form residual

$$
\begin{aligned}
& \boldsymbol{r}_{u}=\boldsymbol{f}-A \boldsymbol{u}^{k}-B^{t} p^{k}, \\
& r_{p}=g-B \boldsymbol{u}^{k}+A_{p} p^{k} .
\end{aligned}
$$

Step 2. Relaxation of velocity $\boldsymbol{u}$ and $p$ by solving momentum equation:

$$
\begin{aligned}
& \boldsymbol{e}_{u}=\mathcal{W}\left(S_{u} \backslash \boldsymbol{r}_{u}\right), \\
& \boldsymbol{u}^{k+\frac{1}{2}}=\boldsymbol{u}^{k}+\boldsymbol{e}_{u}, \\
& p^{k+\frac{1}{2}}=p^{k}+M_{21} \boldsymbol{e}_{u} .
\end{aligned}
$$

Step 3. Form residual and relax a fourth order equation for pressure:

$$
\begin{aligned}
& r_{p}^{1}=g-B \boldsymbol{u}^{k+\frac{1}{2}}+A_{p} p^{k+\frac{1}{2}}-\left(r_{p}-\omega r_{p}\right), \\
& e_{p}=S_{p} \backslash r_{p}^{1} .
\end{aligned}
$$

Step 4. Update $\boldsymbol{u}, p$ :

$$
\begin{aligned}
& \boldsymbol{u}^{k+1}=\boldsymbol{u}^{k+\frac{1}{2}}+M_{12} e_{p}, \\
& p^{k+1}=p^{k+\frac{1}{2}}+M_{22} e_{p} .
\end{aligned}
$$


Remark 3.4. As we have discussed the properties of the matrices $S_{u}$ and $S_{p}$ in the transformed system $\mathcal{T}$ in Remarks 3.2 and 3.3. Effective smoothers for matrix $S_{u}$ and $S_{p}$ are needed. As we know, Gauss-Seidel iterative method works pretty well for the high frequency of the Laplace or vector Laplace operator. Therefore, we use the 2-step GaussSeidel algorithm for the vector Laplace matrix $S_{u}$. Numerical test show that Gauss-Seidel method works very well even though the matrix $S_{u}$ is not exactly discrete matrix of the vector Laplace operator because of the boundary condition.

Remark 3.5. For the fourth order matrix $S_{p}$, direct Gauss Seidel smoother does not perform well. While since matrix $S_{p}$ is approximately the Schur complement matrix of block system $\mathcal{L}$, it determines the total performance of the distributive Gauss Seidel iterative methods. The good news is from the construction of matrix $S_{p}$, we find that $S_{p}$ mainly consists of two matrices that corresponding to two second order Laplace operators. Therefore, we can use the simple Gauss-Seidel algorithm for each matrix. Detail of analysis and the algorithm is described as follows and in Algorithm 3.2.

Since

$$
\begin{aligned}
S_{p} & =\left[M_{v 0}+\delta t \frac{\kappa}{\eta}(\lambda+2 \mu) C_{0}\right] M_{v 0}^{-1} B_{0} \mathcal{G}_{0} \\
& =A_{1} M_{v 0}^{-1} A_{2},
\end{aligned}
$$

where $A_{1}=M_{v 0}+\delta t \frac{\kappa}{\eta}(\lambda+2 \mu) C_{0}, A_{2}=B_{0} \mathcal{G}_{0}$ and $C_{0}$ is the discrete matrix of scalar Laplace operator with boundary condition, $B_{0}, \mathcal{G}_{0}$ are discrete matrix forms of operators - div, grad enforced with boundary condition respectively. Then an effective smoother can be constructed as follows:

Algorithm 3.2. An algorithm for $S_{p} e_{p}=r_{p}^{1}$.

1. Use two step Gauss-Seidel iterative method for system

$$
A_{1} e_{q}=r_{p}^{1}
$$

2. Multiplied by mass matrix:

$$
e_{q}=M_{v 0} e_{q}
$$

3. Use two-step Gauss-Seidel algorithm for system

$$
A_{2} e_{p}=e_{q} .
$$

Since both $A_{1}, A_{2}$ are scalar Laplace operators, standard Gauss-Seidel iteration method is good smoother for these equations. 
Remark 3.6. We here need to emphasize that the parameter $\omega$ in Step 2 in the smoothing procedure is crucial to the convergence property of the distributive Gauss Seidel iterative method and also to the uniform convergence property of the multigrid algorithm. Values of the parameter varies with respect to the regularity of the mesh. For uniform grid, we choose $\mathcal{\omega}=0.5$ in implementation.

We also study the multigrid algorithm as the right preconditioner for GMRes iterative method for the poroelasticity model prolem, i.e., the GMRes method is used for the following system

$$
\left(\begin{array}{cc}
A & B^{t} \\
B & -A_{p}
\end{array}\right)\left(\begin{array}{cc}
A & B^{t} \\
B & -A_{p}
\end{array}\right)_{M G}^{-1}\left(\begin{array}{l}
\tilde{f} \\
\tilde{g}
\end{array}\right)=\left(\begin{array}{l}
f \\
g
\end{array}\right)
$$

where

$$
\left(\begin{array}{c}
\boldsymbol{u} \\
p
\end{array}\right)=\left(\begin{array}{cc}
A & B^{t} \\
B & -A_{p}
\end{array}\right)_{M G}^{-1}\left(\begin{array}{c}
\tilde{f} \\
\tilde{g}
\end{array}\right) \text { and }\left(\begin{array}{cc}
A & B^{t} \\
B & -A_{p}
\end{array}\right)_{\mathrm{MG}}^{-1}\left(\begin{array}{c}
\tilde{f} \\
\tilde{g}
\end{array}\right),
$$

representing applying multigrid algorithm for

$$
\left(\begin{array}{cc}
A & B^{t} \\
B & -A_{p}
\end{array}\right)\left(\begin{array}{l}
\boldsymbol{u} \\
p
\end{array}\right)=\left(\begin{array}{l}
\tilde{f} \\
\tilde{g}
\end{array}\right) .
$$

As we will see in the following numerical experiments, the preconditioned GMRes method is almost uniformly with respect to both discretization size $h$ and parameter $\delta t$.

\section{Numerical experiments}

In this section, we will implement the finite element method as well as the multigrid algorithm for the following poroelasticity equation

$$
\begin{aligned}
& -\mu \boldsymbol{\Delta} \boldsymbol{u}-(\lambda+\mu) \text { graddiv } \boldsymbol{u}+\nabla p=\boldsymbol{f}, \\
& \operatorname{div} \boldsymbol{u}-\delta t \Delta p=g,
\end{aligned}
$$

with homogeneous Dirichlet boundary condition

$$
\boldsymbol{u}=0, \quad p=0, \quad x \in \partial \Omega,
$$

with $\Omega=[-1,1]^{2}$. We choose parameters in (2.10) $\eta=\kappa=\lambda=\mu=1$ for simplicity. Relaxation parameter $\omega$ is chosen as 0.5 in implementation. We test the following two examples, i.e., we choose functions $f, g$ such that the exact solutions of examples are as follows.

Example 4.1. The exact displacement and pore pressure are

$$
\begin{aligned}
& p(x, y)=x^{2}(x-1)^{2} y^{2}(y-1)^{2} \\
& \boldsymbol{u}(x, y)=\left(\begin{array}{l}
\delta t\left(2 x^{2}-2\right)(2 x-1) y^{2}(y-1)^{2} \\
\delta t x^{2}(x-1)^{2}\left(2 y^{2}-2\right)(2 y-1)
\end{array}\right) .
\end{aligned}
$$



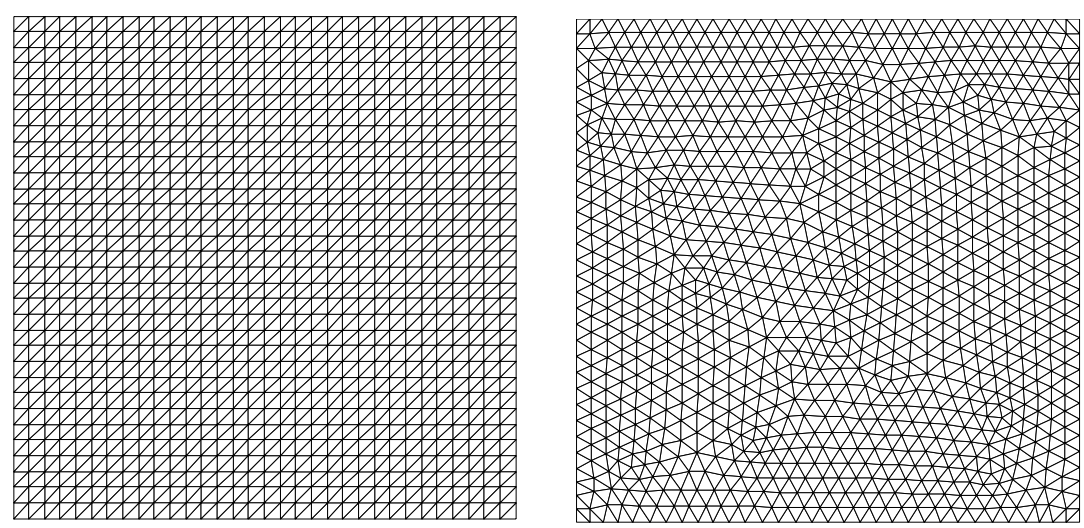

Figure 1: Triangle meshe1 (left) used mesh2 (right) for test.

Example 4.2. The exact displacement and pore pressure are

$$
\begin{aligned}
& p(x, y)=\sin 2 \pi x \sin 2 \pi y, \\
& \boldsymbol{u}(x, y)=\left(\begin{array}{l}
2 \delta t \pi(\cos 2 \pi x-1) \sin 2 \pi y \\
2 \delta t \pi(\cos 2 \pi y-1) \sin 2 \pi x
\end{array}\right) .
\end{aligned}
$$

We only consider the performance of finite element method and multigrid algorithm on a uniform mesh and refine the mesh uniformly. In the triangulation, we mainly choose two kinds of meshes, one is the uniform mesh (mesh1) which is derived by uniformly refining a square, see first figure of Fig. 1 and the second one (mesh2) is almost uniform, see the second figure of Fig. 1. Lowest order Nédélec element is used for displacement variable and piecewise linear finite element is used for pore pressure in our test. For the multigrid algorithm with distributive Gauss Seidel iteration method, we mainly focus on the W-cycle with two-step presmoothing and two-step postsmoothing (W(2,2)-MG) and preconditioned flexible GMRes method with V(2,2)-MG (V-cycle multigrid method with two-step presmoothing and two-step postsmoothing) as preconditioner. Stop criterion of the multigrid algorithm and the preconditioned GMRes method is chosen as relative residual error less than tolerance of $10^{-6}$. In this multigrid algorithm, we mainly concern the iteration numbers as well as the convergence factor to different discretization mesh sizes $h$ and parameter $\delta t$. For the Preconditioned GMRes method, we only present the number of iterations. Finite element package $i$ FEM [28] is used during the implementation.

\subsection{Convergence order of finite element method for the poroelasticity model}

We first present the convergence order of the finite element discretization method. We use Nédélec element for the poroelasticity model problem with different parameter $\delta t$ and uniformly refined the coarse mesh to derive the fine mesh. We first present the error 

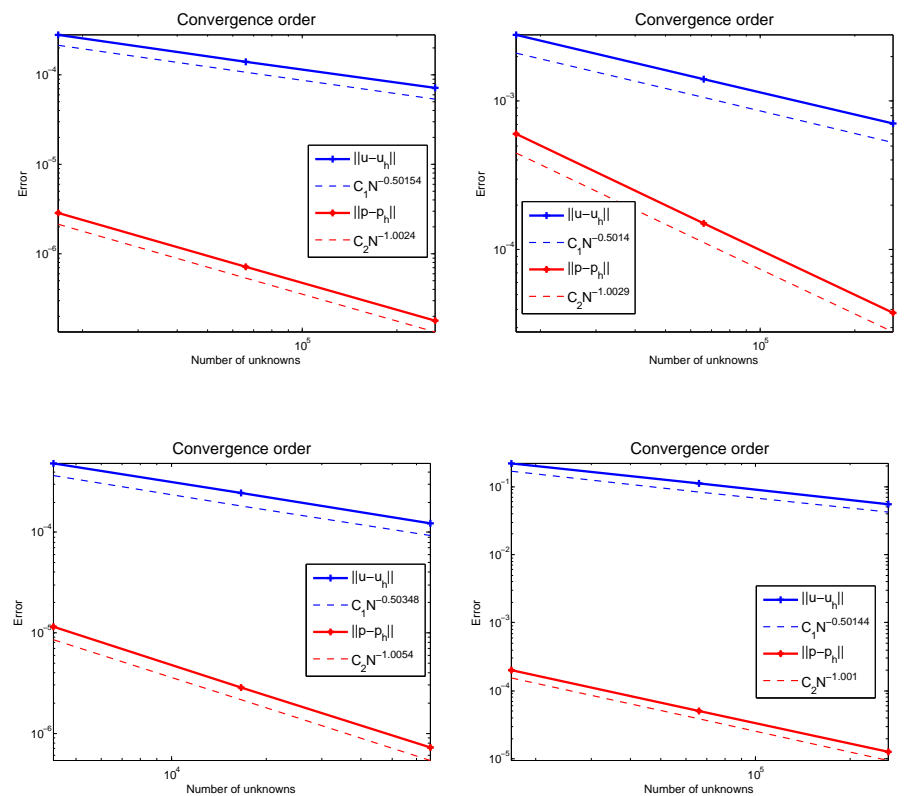

Figure 2: Convergence order in $L^{2}$ for Example 4.1 (left) and Example 4.2 (right) with $\delta t=0.01$ on mesh1 (first row) and mesh2 (second row).

of Example 4.1, Example 4.2 in $L^{2}(\Omega)$ norm on mesh1 and mesh2, then show the convergence order of displacement $\boldsymbol{u}$ in $\boldsymbol{H}$ (rot) norm and pore pressure in energy norm of $H^{1}(\Omega)$ on both meshes. From numerical experiment results in Fig. 2, we can see that both displacement $\boldsymbol{u}$ and pore pressure $p$ have optimal order in $L^{2}(\Omega)$ norm on both meshes, i.e., we have

$$
\left\|\boldsymbol{u}-\boldsymbol{u}_{h}\right\| \lesssim h \text { and }\left\|p-p_{h}\right\| \lesssim h^{2} .
$$

From Fig. 3 , we can also see the convergence order of $\boldsymbol{u}$ in norm $\|\cdot\|_{\text {rot }}$ and $p$ in norm $\|\cdot\|_{1}$ are optimal on the both meshes by this finite element method. On the mesh1. The convergence order of displacement $\boldsymbol{u}$ in $\boldsymbol{H}$ (rot) norm is $\left\|\boldsymbol{u}-\boldsymbol{u}_{h}\right\|_{\text {rot }} \lesssim h$ and pressure $p$ in energy norm of $H^{1}$ is $\left\|p-p_{h}\right\|_{1} \lesssim h$.

\subsection{Performance of multigrid algorithm and preconditioned GMRes method}

In this subsection, we will display the performance of multigrid algorithm as well as the preconditioned flexible GMRes method (without restart) for the poroelasticity problem. Distributive Gauss-Seidel iteration is used as a smoother in the multigrid algorithm. Number of iterations and convergence factor (adjacent ratio of relative residual in bracket) are shown for the multigrid algorithm. We first demonstrate results of the multigrid method with V-cycle for Example 4.1 on both two types of meshes. Dof in the table representing degree of freedom which depends on mesh size $h$ and $*$ in the table means the method does not convergence within 200 iterations. As we will see from the numeri- 

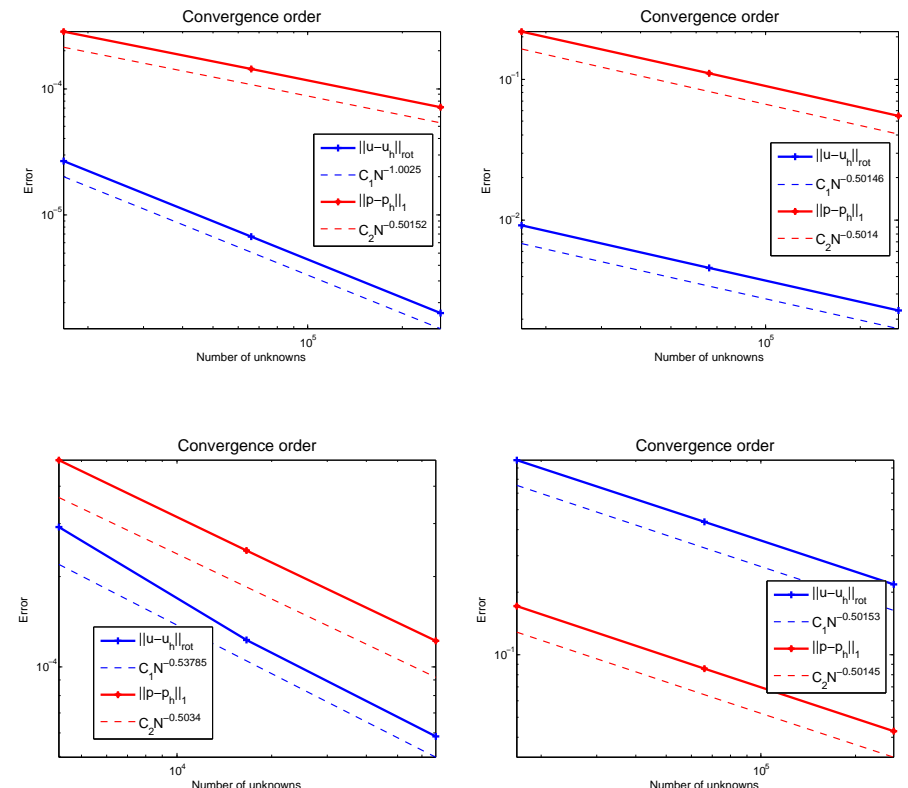

Figure 3: Convergence order in $\boldsymbol{H}(\mathrm{rot})-H^{1}$ for Example 4.1 (left) and Example 4.2 (right) with $\delta t=0.01$ on mesh1 (first row) and mesh2 (second row).

cal results, the V-cycle multigrid method with two-step pre and two-step post smoothing is an almost uniform method for large $\delta t$, see Tables 1,2 . We mainly focus on numerical results of the $\mathrm{W}$-cycle multigrid algorithm as well as the $\mathrm{V}(2,2)-\mathrm{MG}$ preconditioned flexible GMRes method. As we can see from the data in Tables 3, 4 and 5, 6, W-cycle multigrid with both two pre- and post-smoothing is an approximately uniform convergent algorithm to mesh size $h$ on both uniform meshes. We also test the $\mathrm{W}$-cycle multigrid algorithm to different parameters $\delta t$. Numerical results show that number of iterations has a little increase when $\delta t=100,10^{-6}, 10^{-8}$ but is uniform when $\delta t=1,10^{-2}, 10^{-4}$. Therefore, the multigrid method is almost uniformly convergent to the parameter $\delta t$. Same results can also be found for the preconditioned GMRes method, see Tables 7, 8, 9 and 10.

Table 1: V(2,2)-MG with DGS smoother for Example 4.1 w.r.t different parameters $h, \delta t$ on mesh1.

\begin{tabular}{||c|cccccc||}
\hline Dof & $1 \mathrm{e}+2$ & $1 \mathrm{e}-0$ & $1 \mathrm{e}-2$ & $1 \mathrm{e}-4$ & $1 \mathrm{e}-6$ & $1 \mathrm{e}-8$ \\
\hline 16129 & $24(0.41)$ & $18(0.41)$ & $14(0.38)$ & $12(0.31)$ & $24(0.62)$ & $24(0.62)$ \\
\hline 65025 & $27(0.45)$ & $21(0.44)$ & $16(0.42)$ & $13(0.36)$ & $81(0.88)$ & $110(0.90)$ \\
\hline 261121 & $29(0.46)$ & $23(0.46)$ & $17(0.44)$ & $14(0.40)$ & $*$ & $*$ \\
\hline
\end{tabular}


Table 2: V(2,2)-MG with DGS smoother for Example 4.1 w.r.t different parameters $h, \delta t$ on mesh2.

\begin{tabular}{||c|cccccc||}
\hline Dof & $1 \mathrm{e}+2$ & $1 \mathrm{e}-0$ & $1 \mathrm{e}-2$ & $1 \mathrm{e}-4$ & $1 \mathrm{e}-6$ & $1 \mathrm{e}-8$ \\
\hline 16129 & $16(0.28)$ & $12(0.28)$ & $9(0.36)$ & $11(0.27)$ & $24(0.68)$ & $25(0.62)$ \\
\hline 65025 & $17(0.29)$ & $13(0.29)$ & $10(0.29)$ & $12(0.31)$ & $64(0.86)$ & $86(0.89)$ \\
\hline 261121 & $18(0.31)$ & $14(0.40)$ & $11(0.33)$ & $12(0.33)$ & $*$ & $*$ \\
\hline
\end{tabular}

Table 3: W(2,2)-MG with DGS smoother for Example 4.1 w.r.t different parameters $h, \delta t$ on mesh1.

\begin{tabular}{||c|cccccc||}
\hline Dof & $1 \mathrm{e}+2$ & $1 \mathrm{e}-0$ & $1 \mathrm{e}-2$ & $1 \mathrm{e}-4$ & $1 \mathrm{e}-6$ & $1 \mathrm{e}-8$ \\
\hline 16129 & $23(0.39)$ & $18(0.39)$ & $13(0.37)$ & $8(0.18)$ & $15(0.45)$ & $15(0.46)$ \\
\hline 65025 & $25(0.42)$ & $20(0.42)$ & $15(0.39)$ & $8(0.2)$ & $16(0.49)$ & $17(0.52)$ \\
\hline 261121 & $26(0.43)$ & $21(0.43)$ & $16(0.40)$ & $9(0.3)$ & $15(0.45)$ & $19(0.56)$ \\
\hline
\end{tabular}

Table 4: W(2,2)-MG with DGS smoother for Example 4.1 w.r.t different parameters $h, \delta t$ on mesh2.

\begin{tabular}{||c|cccccc||}
\hline Dof & $1 \mathrm{e}+2$ & $1 \mathrm{e}-0$ & $1 \mathrm{e}-2$ & $1 \mathrm{e}-4$ & $1 \mathrm{e}-6$ & $1 \mathrm{e}-8$ \\
\hline 16129 & $15(0.27)$ & $12(0.27)$ & $9(0.26)$ & $7(0.09)$ & $15(0.44)$ & $15(0.46)$ \\
\hline 65025 & $16(0.28)$ & $13(0.28)$ & $10(0.28)$ & $6(0.18)$ & $16(0.47)$ & $17(0.51)$ \\
\hline 261121 & $17(0.29)$ & $13(0.29)$ & $10(0.28)$ & $7(0.25)$ & $14(0.43)$ & $19(0.55)$ \\
\hline
\end{tabular}

Table 5: W(2,2)-MG with DGS smoother for Example 4.2 w.r.t different parameters $h, \delta t$ on mesh1.

\begin{tabular}{||c|cccccc||}
\hline Dof & $1 \mathrm{e}+2$ & $1 \mathrm{e}-0$ & $1 \mathrm{e}-2$ & $1 \mathrm{e}-4$ & $1 \mathrm{e}-6$ & $1 \mathrm{e}-8$ \\
\hline 16129 & $23(0.39)$ & $18(0.41)$ & $14(0.37)$ & $7(0.16)$ & $12(0.45)$ & $12(0.46)$ \\
\hline 65025 & $26(0.42)$ & $20(0.42)$ & $16(0.40)$ & $8(0.23)$ & $12(0.48)$ & $13(0.52)$ \\
\hline 261121 & $26(0.43)$ & $22(0.43)$ & $17(0.41)$ & $10(0.3)$ & $11(0.45)$ & $14(0.56)$ \\
\hline
\end{tabular}

Table 6: W(2,2)-MG with DGS smoother for Example 4.2 w.r.t different parameters $h, \delta t$ on mesh2.

\begin{tabular}{||c|cccccc||}
\hline Dof & $1 \mathrm{e}+2$ & $1 \mathrm{e}-0$ & $1 \mathrm{e}-2$ & $1 \mathrm{e}-4$ & $1 \mathrm{e}-6$ & $1 \mathrm{e}-8$ \\
\hline 16129 & $15(0.27)$ & $12(0.27)$ & $9(0.26)$ & $6(0.08)$ & $11(0.44)$ & $12(0.46)$ \\
\hline 65025 & $16(0.28)$ & $13(0.28)$ & $10(0.28)$ & $6(0.19)$ & $12(0.47)$ & $13(0.51)$ \\
\hline 261121 & $17(0.29)$ & $14(0.29)$ & $11(0.28)$ & $7(0.24)$ & $11(0.43)$ & $14(0.55)$ \\
\hline
\end{tabular}

\section{Conclusions}

In this work, we investigate multigrid algorithm with distributive Gauss Seidel relaxation for poroelasticity model problem by finite element method with homogeneous boundary 
Table 7: GMRes with preconditioner V(2,2)-MG for Example 4.1 w.r.t different parameters $h$, $\delta t$ on mesh1.

\begin{tabular}{||c|cccccc||}
\hline Dof & $1 \mathrm{e}+2$ & $1 \mathrm{e}-0$ & $1 \mathrm{e}-2$ & $1 \mathrm{e}-4$ & $1 \mathrm{e}-6$ & $1 \mathrm{e}-8$ \\
\hline 16129 & 14 & 12 & 10 & 9 & 11 & 11 \\
\hline 65025 & 17 & 14 & 12 & 10 & 14 & 14 \\
\hline 261121 & 19 & 15 & 13 & 11 & 16 & 17 \\
\hline
\end{tabular}

Table 8: GMRes with preconditioner V(2,2)-MG for Example 4.1 w.r.t different parameters $h$, $\delta t$ on mesh2.

\begin{tabular}{|c|cccccc||}
\hline Dof & $1 \mathrm{e}+2$ & $1 \mathrm{e}-0$ & $1 \mathrm{e}-2$ & $1 \mathrm{e}-4$ & $1 \mathrm{e}-6$ & $1 \mathrm{e}-8$ \\
\hline 16129 & 10 & 8 & 7 & 8 & 10 & 10 \\
\hline 65025 & 11 & 9 & 8 & 9 & 12 & 13 \\
\hline 261121 & 13 & 10 & 9 & 9 & 14 & 15 \\
\hline
\end{tabular}

Table 9: GMRes with preconditioner V(2,2)-MG for Example 4.2 w.r.t different parameters $h$, $\delta t$ on mesh1.

\begin{tabular}{||c|cccccc||}
\hline Dof & $1 \mathrm{e}+2$ & $1 \mathrm{e}-0$ & $1 \mathrm{e}-2$ & $1 \mathrm{e}-4$ & $1 \mathrm{e}-6$ & $1 \mathrm{e}-8$ \\
\hline \hline 16129 & 14 & 12 & 10 & 9 & 12 & 12 \\
\hline 65025 & 17 & 13 & 12 & 11 & 14 & 15 \\
\hline 261121 & 19 & 15 & 13 & 12 & 17 & 18 \\
\hline
\end{tabular}

Table 10: GMRes with preconditioner V(2,2)-MG for Example 4.2 w.r.t different parameters $h$, $\delta t$ on mesh2.

\begin{tabular}{||c|cccccc||}
\hline Dof & $1 \mathrm{e}+2$ & $1 \mathrm{e}-0$ & $1 \mathrm{e}-2$ & $1 \mathrm{e}-4$ & $1 \mathrm{e}-6$ & $1 \mathrm{e}-8$ \\
\hline 16129 & 11 & 8 & 8 & 8 & 11 & 11 \\
\hline 65025 & 12 & 9 & 9 & 9 & 14 & 14 \\
\hline 261121 & 13 & 11 & 10 & 10 & 16 & 17 \\
\hline
\end{tabular}

conditions. Nédélec edge element and piecewise continuous Lagrange polynomial are used for the displacement and pressure variables of the model problem. Numerical experiments show that the finite element discretization method achieves optimal order and the constructed multigrid method is uniformly convergent to both mesh sizes $h$ and parameter $\delta t$. In our future work, we will make a theoretically convergence and stability analysis for the finite element method as well as the multigrid algorithm.

\section{Acknowledgements}

The first author is supported by the National Natural Science Foundation of China under Grant No. 11501473, No. 11426189 and the Fundamental Research Funds for the Central Universities of China (No. 2682016CX108). The second author is supported by the 
National Natural Science Foundation of China under Grant No. 11671157.

\section{References}

[1] J. H. Adler, F. J. Gaspar, X. HU, C. ROdRIgo And L. T. Zikatanov, Robust block preconditioners for Biot's model, Arxive, (2017), pp. 1-13.

[2] R. AsADI, B. ATAIEASHTIANI AND C. T. SimmOns, Finite volume coupling strategies for the solution of a biot consolidation model, Comput. Geotech., 55 (2014), pp. 494-505.

[3] T. Baerland, J. J. LeE, K. A. MARDAl AND R. Winther, Weakly imposed symmetry and robust preconditioners for biot's consolidation model, Comput. Methods Appl. Math., 17 (2017).

[4] M. A. Biot, General theory of three-demensional conslidation, J. Appl. Phys., 12(2) (1994), pp. $155-164$.

[5] M. A. BIOT, General solutions of the equations of elasticity and Consolidation for porous material, J. Appl. Mech., (1958).

[6] S. H. Chan, K. K. Phoon And F. H. Lee, A modified Jacobi preconditioner for solving illconditioned Biot's consolidation equations using symmetric quasi-minimal residual method, Int. J. Numer. Anal. Methods Geomechan., 25(10) (2001), pp. 1001-1025.

[7] F. J. GASPAR, J. L. GRACIA, F. J. LisbONA AND P. N. VABISHCHEVICH, A stabilized method for a secondary consolidation Biot's model, Numer. Methods Partial Differential Equations, (2007).

[8] F. J. Gaspar, F. J. Lisbona, C. W. Oosterlee and P. N. Vabishchevich, An efficient multigrid solver for a reformulated version of the poroelasticity system, Comput. Methods Appl. Mech. Eng., 196(8) (2007), pp. 1447-1457.

[9] F. J. Gaspar, F. J. Lisbona, C. W. OOsterlee And R. Wienands, A systematic comparison of coupled and distributive smoothing in multigrid for the poroelasticity system, Numer. Linear Algebra Appl., 11(23) (2004), pp. 93-113.

[10] X. HU, L. MU AND X. YE, Weak Galerkin method for the Biot's consolidation model, Comput. Math. Appl., (inpress), (2017).

[11] J. J. LeE, K. A. MARDAL AND R. WINTHER, Parameter-robust discretization and preconditioning of biot's consolidation model, SIAM J. Sci. Comput., 39(1) (2015), pp. A1-A24.

[12] A. F. MAK, L. HUANG AND Q. WANG, A biphasic poroelastic analysis of the flow dependent subcutaneous tissue pressure and compaction due to epidermal loadings: issues in pressure sore, J. Biomechan. Eng., 116(4) (1994), pp. 421.

[13] A. M. Marcio, T. Vidar AND A. F. D. Loula, Asymptotic behavior of semidiscrete finiteelement approximations of Biot's consolidation problem, SIAM J. Numer. Anal., 33(3) (1996), pp. 1065-1083.

[14] J. P. Morris, Y. HaO, W. Foxall AND W. MCNAB, A study of injection-induced mechanical deformation at the in salah $\mathrm{Co}_{2}$ storage project, Int. J. Greenhouse Gas Control, 5(2) (2011), pp. 270-280.

[15] V. C. Mow, M. K. KWAN, W. M. Lai AND M. H. Holmes, A finite deformation theory for nonlinearly permeable soft hydrated biological tissues, Front. Biomech., (1986), pp. 153-179.

[16] M. A. MurAD AND A. F. D. Loula, Improved accuracy in finite element analysis of Biot's consolidation problem, Comput. Methods Appl. Mech. Eng., 95(3) (1992), pp. 359-382.

[17] P. J. PHILliPS AND M. F. WHEELER, A coupling of mixed and continuous Galerkin finite element methods for poroelasticity I: the continuous in time case, Comput. Geosci., 11(2) (2007), pp. 131144. 
[18] P. J. PHILliPs AND M. F. WHEELER, A coupling of mixed and continuous Galerkin finite element methods for poroelasticity II: the discrete-in-time case, Comput. Geosci., 11(2) (2007), pp. 145-158.

[19] P. J. Phillips AND M. F. WHEeler, A coupling of mixed and discontinuous Galerkin finiteelement methods for poroelasticity, Comput. Geosci., 12(4) (2008), pp. 417-435.

[20] B. R. Simon, M. V. Kaufmann, M. A. McAfee And A. L. BALDWin, Finite element models for arterial wall mechanics, J. Biomech. Eng., 115(4B) (1993), pp. 489-496.

[21] Maria Tchonkova, JOHn Peters And Stein Sture, A new mixed finite element method for poro-elasticity, Int. J. Numer. Anal. Methods Geomech., 32(6) (2010), pp. 579-606.

[22] K. TERZAGHI, Erdbaumechanik auf Bodenphysikalischer Grundlage, 1925.

[23] AlEXANDER Z̆ENIŠEK, The existence and uniqueness theorem in Biot's consolidation theory, Aplikace Matematiky, 29(3) (1984), pp. 194-211.

[24] R. WienAnds, F. J. GASPAR, F. J. LisBOnA AND C. W. OOSTERLEe, An efficient multigrid solver based on distributive smoothing for poroelasticity equations. Comput., 73(2) (2004), pp. 99119.

[25] M. YANG AND L. A. TABER, The possible role of poroelasticity in the apparent viscoelastic behavior of passive cardiac muscle, J. Biomech., 24(7) (1991), pp. 587-597.

[26] S. Y. YI, Convergence analysis of a new mixed finite element method for Biot's consolidation model, Numer. Methods Partial Differential Equasion, 23(11,2013) (2007), pp. 904-922.

[27] S. Y. YI, A coupling of nonconforming and mixed finite element methods for Biot's consolidation model, Numer. Methods for Partial Differential Equations, 29(5) (2013), pp. 1749-1777.

[28] L. CHEN, iFEM: An integrated finite element methods package in MATLAB, Technical Report, 2009. 